\title{
RESEARCH
}

\section{miR-183-5p regulates uterine receptivity and enhances embryo implantation}

\author{
Rubab Akbar1,2,*, Kamran Ullah',2,3,*, Tanzil Ur Rahman'1,2, Yi Cheng1,2, Hai-Yan Pang1,2, Lu-Yang Jin 1,2, Qi-Jing Wang1,4, \\ He-Feng Huang 1,5 and Jian-Zhong Sheng1,2 \\ 1The Key Laboratory of Reproductive Genetics (Zhejiang University), Ministry of Education, Hangzhou, Zhejiang, China \\ 2Department of Pathology and Pathophysiology, School of Medicine, Zhejiang University, Hangzhou, Zhejiang, China \\ ${ }^{3}$ Department of Zoology, University of Swabi, Anbar, Khyber Pakhtunkhwa, Pakistan \\ 4Department of Reproductive Endocrinology, School of Medicine, Zhejiang University, Hangzhou, Zhejiang, China \\ 5The International Peace Maternity and Child Health Hospital, School of Medicine, Shanghai Jiao Tong University, Shanghai, China
}

Correspondence should be addressed to H-F Huang or J-Z Sheng: huanghefg@hotmail.com or shengjz@zju.edu.cn

*(R Akbar and K Ullah contributed equally to this work)

\begin{abstract}
Receptive endometrium is a prerequisite for successful embryo implantation, and it follows that poor endometrial receptivity is a leading cause of implantation failure. miRNAs play important roles as epigenetic regulators of endometrial receptivity and embryo implantation through post-transcriptional modifications. However, the mechanisms of action of many miRNAs are poorly understood. In this study, we investigated the role of the miR-183 family, comprising three miRNAs (miR-183-5p, miR-182-5p, and miR-96-5p) in endometrial receptivity and embryo implantation. The miR-183 family shows estrogen-dependent upregulation in endometrial Ishikawa (IK) cells. The miR-183 family also has a positive role in migration and proliferation of IK cells. Furthermore, JAr spheroid attachment experiments show that attachment rates were significantly decreased after treatment of IK cells with inhibitors for miR-183-5p and miR-182-5p and increased after treatment with miR-183-5p-mimic and miR-96-5p-mimic, respectively. The downstream analysis shows that catenin alpha 2 (CTNNA2) is a potential target gene for miR-183-5p, and this was confirmed in luciferase reporter assays. An in vivo mouse pregnancy model shows that inhibition of miR-183-5p significantly decreases embryo implantation rates and increases CTNNA2 expression. Downregulation of CTNNA2 in endometrial cells by miR-183-5p may be significant in mediating estrogenic effects on endometrial receptivity. In conclusion, miR-183-5p and the CTNNA2 gene may be potential biomarkers for endometrial receptivity and may be useful diagnostic and therapeutic targets for successful embryo implantation.
\end{abstract}

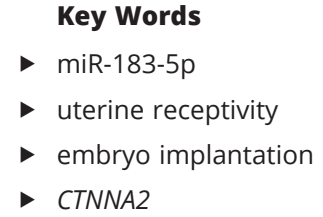

Journal of Molecular Endocrinology (2020) 64, 43-52

\section{Introduction}

Endometrial receptivity is critical for successful embryo implantation. It refers to the restricted period when the uterus acquires a suitable morphological and functional state, compatible with blastocyst adhesion and implantation. This period spanning between 20 and
24 days of the human menstrual cycle is often described as the 'window of implantation' (Strowitzki et al. 2006). During the process of implantation, an intimate connection is established between the blastocyst and maternal endometrial surface, forming the placenta and 
providing a connection between the growing fetus and maternal circulation. For successful embryo implantation, a healthy, functional embryo at the blastocyst stage and receptive maternal endometrium are necessary (Achache \& Revel 2006). Recent studies show that embryo implantation and endometrial receptivity are regulated by classic transcriptional mechanisms and several other epigenetic phenomena, including DNA methylation, histone modification and small non-coding RNA (miRNA) modifications (Bartel 2004, Ha \& Kim 2014).

The miRNA molecules of approximately 20-22 nucleotides have important roles in RNA silencing and post-transcriptional gene regulation. Several studies have reported their roles in embryo implantation (Bartel 2004, Ha \& Kim 2014). The discovery of extracellular miRNAs opened up a new avenue for implantation studies (Liang et al. 2017). Recent studies show that miRNAs play protagonist roles in cellular processes including cellular differentiation, proliferation, migration, and apoptosis (Liang et al. 2017). Several studies have reported different roles of miRNAs in uterine receptivity. Zheng et al. (2017) found that miR-200c may impair uterine receptivity and maybe useful as a biomarker for clinical application. Yang et al. (2018) showed that miR-543 was associated with endometrial receptivity, and its downregulation might affect embryo implantation. The miR-183 family, comprising three miRNAs (miR-183-5p, miR-182-5p, and miR-96-5p) with sequence homology, has been reported in the human uterus (Estella et al. 2012, Vilella et al. 2015); however, its target role in endometrial receptivity and the ultimate effect on embryo implantation remains unclear.

In this study, we explored the role of the miR-183 family in endometrial receptivity and implantation. We found that miR-183-5p could regulate alpha catenin 2 gene (CTNNA2) that might mediate endometrial receptivity. CTNNA2 is a cytoskeleton protein responsible for cell-cell adhesion, and it negatively regulates the Wnt signaling pathway (Gates \& Peifer 2005, Sun et al. 2014). Exploring the function of the miR-183 family and their potential target genes in endometrial receptivity and embryo implantation may improve our understanding of the respective mechanisms.

\section{Materials and methods}

\section{Cell culture}

This research study was approved by the research and ethical committee of the Women's Hospital, Zhejiang University, Hangzhou, China. Ishikawa (IK) cells, human endometrial cell line (American Type Culture Collection) were cultured using RPMI-1640 medium supplemented with $10 \%$ fetal bovine serum (FBS; $\mathrm{vol} / \mathrm{vol}), 100 \mathrm{U} / \mathrm{mL}$ streptomycin and $100 \mathrm{U} / \mathrm{mL}$ penicillin at $37^{\circ} \mathrm{C}$ plus $5 \% \mathrm{CO}_{2}$.

\section{Transfection of IK cells with miRNA inhibitors and mimics}

The inhibitors for miRs are chemically synthesized, complementary, antisense, single-stranded oligonucleotides to their target: endogenous, mature miRNA (Bai et al. 2017). Inhibitors for miR-183-5p (I-miR183-5p), miR-182-5p (I-miR-182-5p) and miR-96-5p (I-miR-96-5p), and negative controls were manufactured by Applied Biological Materials Inc. (abm), Richmond, BC, Canada. Mimics for miR-183-5p (miR-183-5pmimic), miR-182-5p (miR-182-5p-mimic) and miR-96-5p (miR-96-5p-mimic), and negative controls were obtained from Genepharma (Shanghai, China). The sequences of inhibitors and mimics are shown in Supplementary Table 1 (see section on supplementary materials given at the end of this article). Negative controls (NCs) were validated by random sequences, tested on mammalian cells and tissues, and shown to produce no identifiable effects on known miR function.

Before transfection, IK cells were cultured in normal medium containing estradiol $\left(\mathrm{E}_{2}\right)$ (Sigma) for $48 \mathrm{~h}$. In the inhibition experiments, IK cells in each group were treated with negative control and $10^{-7} \mathrm{M} \mathrm{E}_{2}\left(\mathrm{NC}+10^{-7} \mathrm{M}\right.$ $\mathrm{E}_{2}$ group), I-miR-183-5p and 10-7 $\mathrm{M} \mathrm{E}_{2}$ (I-miR-183-5p+10-7 $\mathrm{M} \mathrm{E} \mathrm{E}_{2}$ group), I-miR-182-5p and $10^{-7} \mathrm{M} \mathrm{E}_{2}$ (I-miR$182-5 p+10^{-7} \mathrm{M} \mathrm{E}_{2}$ group), and, I-miR-96-5p and $10^{-7} \mathrm{M}$ $\mathrm{E}_{2}$ (I-miR-96-5p+10-7 $\mathrm{M} \mathrm{E}_{2}$ group), respectively. In the mimic experiments, IK cells in each group were treated with negative control and $10^{-9} \mathrm{M} \mathrm{E}_{2}\left(\mathrm{NC}+10^{-9} \mathrm{M} \mathrm{E}_{2}\right.$ group), miR-183-5p-mimics and 10-9 $\mathrm{M} \mathrm{E}_{2}$ (miR-183-5pmimic $+10^{-9} \mathrm{M} \mathrm{E}_{2}$ group), miR-182-5p-mimic and $10^{-9} \mathrm{M}$ $\mathrm{E}_{2}$ (miR-182-5p-mimic $+10^{-9} \mathrm{M} \mathrm{E}_{2}$ group), and miR-965p-mimic and 10-9 $\mathrm{M} \mathrm{E}_{2}$ (miR-96-5p mimic $+10^{-9} \mathrm{M} \mathrm{E}_{2}$ group), respectively. IK cells were also treated with $10^{-9}$ $\mathrm{M} \mathrm{E}_{2}$ alone (10-9 group) and $10^{-7} \mathrm{M} \mathrm{E}_{2}$ alone $\left(10^{-7} \mathrm{M} \mathrm{E}_{2}\right.$ group) respectively in order to verify the estrogenic effects.

When cells reached 70-90\% confluence, cells were transfected with $5 \mathrm{nM}$ inhibitors, $5 \mathrm{nM}$ mimics and their negative controls respectively using RNAifectin ${ }^{\mathrm{TM}}$ (Cat. No. G073) according to the manufacturer's guidelines (Applied Biological Materials Inc.). After transfection, the transfection medium was substituted with normal medium and cultured for different intervals based on 
the purposes of different experiments. Protein and RNA were collected for Western blot and real-time PCR (Q-PCR) analyses.

\section{Quantitative real-time PCR}

After transfection and subsequent incubation, IK cells were scraped using cell scraper and TRIzol was added. Cell suspension from each well was collected in Eppendorf tubes, and total miRNA extraction was undertaken using phenol chloroform extraction method. RT to cDNA was performed using Mir-X ${ }^{\mathrm{TM}}$ miRNA First-Strand Synthesis kit, according to manufacturer's guidelines (Clontech Laboratories, Inc.). cDNA synthesis reaction contained mRQ buffer $(5 \mu \mathrm{L})$, mRQ enzyme $(1.25 \mu \mathrm{L})$ and RNA sample $(3.75 \mu \mathrm{L}, 0.25-8 \mu \mathrm{g})$, making the total volume $10 \mu \mathrm{L}$. Incubation was performed in thermocycler for $1 \mathrm{~h}$ at $37^{\circ} \mathrm{C}$ followed by termination at $85^{\circ} \mathrm{C}$ for $5 \mathrm{~min}$ to inactivate the enzyme. Then $90 \mu \mathrm{L} \mathrm{ddH}_{2} \mathrm{O}$ was added to make the final volume $100 \mu \mathrm{L}$. Q-PCR was performed using Mir-X miRNA qRT-PCR SYBR Kit (Cat. No. 638314, Clontech Laboratories). Q-PCR reactions were carried out in $10 \mu \mathrm{L}$ reaction mixture containing $1 \mu \mathrm{L}$ of cDNA sample and master mix containg $\mathrm{ddH}_{2} \mathrm{O}(3.6 \mu \mathrm{L})$, SYBR premix $(5 \mu \mathrm{L})$, miR-specific primer $(0.2 \mu \mathrm{L})$ and mRQ $3^{\prime}$ primer $(0.2 \mu \mathrm{L})$. The quantitative Q-PCR analysis was carried out in an Applied Biosystems 7500 Fast (ABI) according to SYBR® qRT-PCR manufacture's guidelines. The expression levels were quantified by measuring cycle threshold $(\mathrm{Ct})$ values and normalized to U6-snRNA (endogenous control for miRNA detection) or GAPDH (endogenous control for mRNA) using the $2^{-\Delta \Delta \mathrm{ct}}$ method. The sequence of primers is given in Supplementary Table 2.

\section{JAr spheroid attachment to IK cells monolayer}

Multicellular spheroids of human choriocarcinoma cells (HTB 144, American Type Culture Collection) (Kolibianakis et al. 2003) were applied to IK cells monolayer. The IK cells were pretreated with $10^{-9} \mathrm{M}$ or $10^{-7} \mathrm{M} \mathrm{E}_{2}$, cultured for $48 \mathrm{~h}$ followed by transfection using miRNA inhibitors or mimics for miR-183-5p, miR-182-5p, and miR-183-5p. JAr spheroids were prepared according to a standard procedure (Hohn et al. 2000) and transferred onto the surface of confluent IK cells monolayer for $1 \mathrm{~h}$ (100 spheroids/well in 24-well plates). Non-adherent spheroids were detached by centrifugation of the plate at $10 \boldsymbol{g}$ for $10 \mathrm{~min}$, keeping cell surface facing down. JAr spheroids were counted under a light microscope and the attachment rate was calculated for each well as follows: Attachment rate $=$ ratio of the number of spheroids attached to the number of spheroids seeded. The experiment was repeated at least three times.

\section{Cell migration assay}

IK cells were cultured in six-well plates. In the inhibition experiments, after transfection, cells were wounded by removing a 500 to $700 \mu \mathrm{m}$ wide strip with a standard $200 \mu \mathrm{L}$ pipette tip and photographed, designated as time $0 \mathrm{~h}$. The cells were then incubated for another $24 \mathrm{~h}$ and were photographed. The distance of migration was calculated using ImageJ software (National Institutes of Health).

\section{Cell proliferation assay}

The confluent cultured IK cells were trypsinized and suspended in RPMI-1640 medium with 10\% FBS. The cells $\left(1 \times 10^{4} / \mathrm{cm}^{2}\right)$ were plated in 96-well plates. After each treatment, cell counting Kit-8 (CCK-8) (Dojindo, Kumamoto, Japan) (Gao et al. 2018) was used to examine viable cells and proliferation rates. The solution of CCK-8 (10 $\mu \mathrm{L}$ ) was added to each well. After $1-4 \mathrm{~h}$ the absorbance (OD value) was measured at $450 \mathrm{~nm}$ using micro-plate reader. The OD value at $450 \mathrm{~nm}$ is proportional to the number of viable cells and proliferation. Each group was in triplicate, so the average OD value for each group was taken.

\section{Target gene prediction}

Target gene prediction was performed using online Target predicting software including Target scan (www. targetscan.org) and miRDB (www.mirbase.org).

\section{Luciferase reporter assay}

The human 3'-UTR of CTNNA2 gene was cloned downstream of hRluc (humanized Renilla luciferase) gene into REPORT ${ }^{\mathrm{TM}}$ Dual-Luciferase miRNA Target Expression Vector (RIBBIO, Guangzhou, China), and a mutant plasmid was constructed with site-directed mutagenesis in the seed region for miR-183-5p. Firefly luciferase gene was used as a normalization reporter within the same plasmid backbone. 293T cells were seeded into a 96-well plate at $1.5 \times 10^{4}$ cells/well and allowed to adhere overnight. Cells were co-transfected with pmiR-RB-REPORT ${ }^{\mathrm{TM}}$ luciferase reporter plasmids (50 ng/well) (RIBBIO) and hsa-miR183-5p mimic (final concentration at $50 \mathrm{nM}$ ) using Lipofectamine 3000 (0.225 $\mu \mathrm{L} /$ well) (RIBBIO) following 
Lipofectamine 3000 Reagent Protocol. The medium was removed at $48 \mathrm{~h}$ post transfection. PBS $(35 \mu \mathrm{L})$ and an equal volume of Dual-Glo ${ }^{\circ}$ Luciferase Reagent were added to each well, mixed and shaken at $25^{\circ} \mathrm{C}$ for $10 \mathrm{~min}$. The mixture was transferred into 96-well LUMITRAC ${ }^{\text {тM }}$ 200 white microplates, and the firefly luminescence was measured. Stop \&Glo® Reagent $(35 \mu \mathrm{l}$, RIBBIO) was replenished in each well, mixed and shaken at $25^{\circ} \mathrm{C}$ for $10 \mathrm{~min}$. The Renilla luminescence was measured.

\section{Western blot analysis}

Tissue lysis was done using RIPA lysis buffer containing protease inhibitor cocktail. Protein concentrations were determined using the BCA kit (Thermo Scientific). Samples at $20 \mu \mathrm{g} / \mathrm{lane}$ were separated on a $10 \%$ sodium dodecyl sulfate-polyacrylamide gel and transferred to nitrocellulose membrane (Schleicher \& Schuel Bioscience $\mathrm{GmbH}$, Dassel, Germany). After blocking in 5\% nonfat milk for $2 \mathrm{~h}$, the membranes were incubated with primary antibodies including rabbit monoclonal antiCTNNA2 antibody (1:2000, Cat\# ab76015, Abcam Inc.), rabbit monoclonal anti-GSK3 $\beta$ antibody (1:5000; Cat\# ab32391, Abcam), and, rabbit monoclonal $\beta$-Actin antibody (1:5000; Santa Cruz Biotechnology) at $4^{\circ} \mathrm{C}$ overnight. After several washes with Tris-buffered saline and $0.2 \%$ Tween 20 , the membranes were incubated with the appropriate secondary antibody for $1 \mathrm{~h}$ at room temperature. Protein intensities were determined and analyzed using Odyssey® Imager (LI-COR, Lincoln, NE, USA). The relative protein levels were quantified using Image $J$ software and were normalized to GAPDH or $\beta$-actin.

\section{Animal experiments}

All animal protocols were approved by the Animal Care and Use Committee, School of Medicine, Zhejiang University. Female ICR mice aged $6-8$ weeks in estrus were bred with male ICR mice aged 8-10 weeks, and the day when vaginal plugging was observed was considered as day 1 of pregnancy. At day 3, pregnant mice were anesthetized at 09:00 $\mathrm{h}$, and, $10 \mu \mathrm{L}$ solution containing $1 \mu \mathrm{g}$ miR-183-5p inhibitor (GenePharma) was injected into the right horn of uterus of each mouse, while the left horn was injected with normal saline. Mice were dissected at day 7 of pregnancy to observe the embryo implantation rate, and, uterine endometrium samples were collected for protein and miRNA analyses.

\section{Statistical analysis}

GraphPad Prism 6 (GraphPad Software) was used for statistical analysis. Student $t$-test was used to determine the significant difference between two groups. All experiments were performed in triplicate. Differences at $P<0.05$ were considered significant.

\section{Results}

miR-183 family is upregulated in IK cells by estrogen

We examined the expression levels of the miR-183 family including miR-183-5p, miR-182-5p and miR96-5p in IK cells treated with different concentrations of $\mathrm{E}_{2}$ for $48 \mathrm{~h} \cdot 1^{-9} \mathrm{M} \mathrm{E}_{2}$ is a basic and physiological concentration, whereas $10^{-7} \mathrm{M} \mathrm{E}_{2}$, a high concentration, could be observed in the period of embryo implantation and pregnancy (Abbassi-Ghanavati et al. 2009, Berkane et al. 2017). Our results showed that, compared to $10^{-9} \mathrm{M} \mathrm{E}_{2}$, treatment of IK cells with $10^{-7} \mathrm{M} \mathrm{E}_{2}$ significantly upregulated expression levels of miR-183-5p, miR-182-5p and-miR-96-5p (Fig. 1).

\section{miR-183 family mediates estrogenic effects on migration and proliferation of IK cells}

Compared to $10^{-9} \mathrm{M} \mathrm{E}_{2}$, treatment of IK cells with $10^{-7} \mathrm{M}$ $\mathrm{E}_{2}$ significantly increased migration (Fig. 2A and D) and proliferation (Fig. 2E) of IK cells. Treatment of IK cells with the miR-183 family inhibitors including I-miR-183-5p, I-miR-182-5p and I-miR-96-5p significantly attenuated the effects of $10^{-7} \mathrm{E}_{2}$ on migration (Fig. $2 \mathrm{~A}$ and B) and
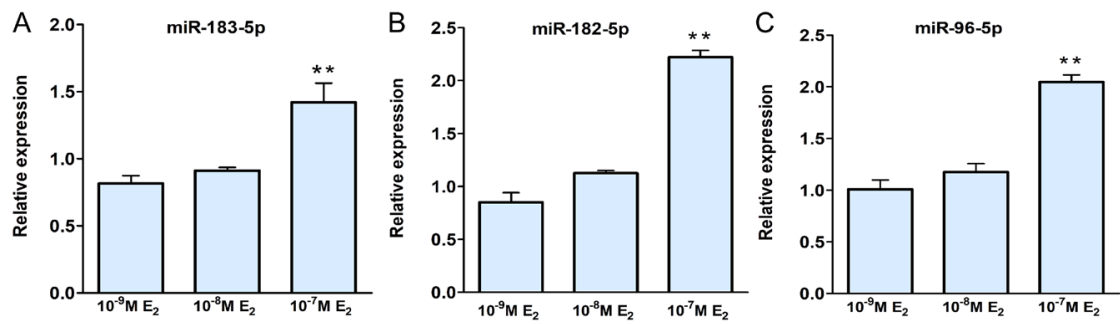

Figure 1

IK cells were treated with different concentrations of estradiol $\left(E_{2}\right)(\mathrm{mol} / \mathrm{L})$. $E_{2}$ dose dependently increased the expression levels of miR-183-5p (A), miR-182-5p (B), and miR-96-5p (C) in IK cells. $* * P<0.01$, compared with control $\left(10^{-9} \mathrm{E}_{2}\right)$. Number of repeated experiments $(n)=3$. 
proliferation (Fig. 2C). Contrary to inhibition, treatment of IK cells with the miR-183 family mimics including miR-183-5p-mimic, and miR-96-5p-mimic but not miR-182-5p-mimic, significantly promoted migration (Fig. 3A and B). Cell proliferation was also promoted by the three mimics in the presence of $10^{-9} \mathrm{M} \mathrm{E}_{2}$ (Fig. 3C). These results suggest that the miR-183 family may play an important role in regulating the migration and proliferative capacity of IK cells by estrogen.

\section{miR-183 family improves JAr spheroid attachment process}

To verify that the endometrial miR-183 family mediates embryo implantation, a JAr-endometrial cell model was deployed in further experiments. Multiple JAr spheroids were added to the IK cell monolayer, and, the attachment rates were calculated (Fig. 4A, B and C). We found that, compared to $10^{-9} \mathrm{M} \mathrm{E}_{2}$, treatment of IK cells with $10^{-7} \mathrm{M} \mathrm{E}_{2}$, significantly increased JAr attachment rates (Fig. 4F), as we have previously reported (Zou et al. 2011, Ullah et al. 2017). Inhibition of miR-183-5p and miR-182-5p, but not miR-96-5p, decreased the JAr attachment rates in the presence of $10^{-7} \mathrm{M} \mathrm{E}_{2}$ (Fig. 4D). However, treatment of IK cells with miR-183-5p-mimic and miR-96-5p-mimic, but not miR-182-mimic, significantly increased the JAr attachment rates in the presence of $10^{-9} \mathrm{M} \mathrm{E}_{2}$ (Fig. 4E).
These findings suggest that the miR-183 family may play an important role in embryo implantation.

\section{CTNNA2 is a potential target gene of miR-183-5p}

As the miR-183 family may play a significant role in both endometrial receptivity and embryo implantation, we tried to predict the target genes of this miRNA family by using bioinformatics software as shown in 'Materials and methods'. CTNNA2 (Catenin Alpha 2) was predicted as one of the target genes regulated by miR-183 family, and previous study showed that CTNNA2 expression levels were significantly different between the receptive and non-receptive phase of the female reproductive cycle (Carson et al. 2002, Horcajadas et al. 2006, Díaz-Gimeno et al. 2011).

We found that, compared to $10^{-9} \mathrm{M} \mathrm{E}_{2}, 10^{-7} \mathrm{M} \mathrm{E}_{2}$ significantly downregulated the expression of CTNNA2 (Fig. 5A). To further verify the binding of miR-183 to the 3'-UTR of CTNNA2 mRNA, we cloned the wild-type (WT) 3'-UTR and mutant-type (MUT) 3'-UTR target sequences into the luciferase reporter vector (pmiR-RB-REPORT ${ }^{\mathrm{TM}}$ vector). The sequences of wild-type and mutant 3'-UTR of CTNNA2 constructs are shown in Supplementary Table 3. Binding of miR-183-5p to its seed sequence within the miRNA recognition element (MRE) at the 3 '-UTR of CTNNA2 mRNA is indicated in Fig. 5B. The luciferase



\section{Figure 2}

Effects of miR-183-5p, miR-182-5p and miR-96-5p inhibitors on cell migration and proliferation of IK cells. $\mathrm{E}_{2}$ at $10^{-7} \mathrm{M}$ significantly increased cell migration (A and D) and proliferation (E). Treatment of IK cells with inhibitors (I-miR183-5p, I-miR182-5p and I-miR96-5p) significantly reduced 10-7 M $E_{2}$-induced cell migration (A and B) and cell proliferation (C). $* P<0.05$ and $* \star P<0.01$, compared with corresponding controls. Number of repeated experiments $(n)=3$. 

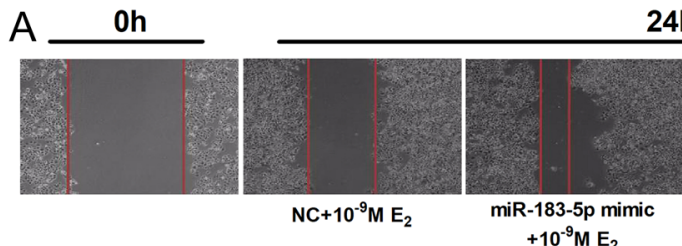

$24 \mathrm{~h}$
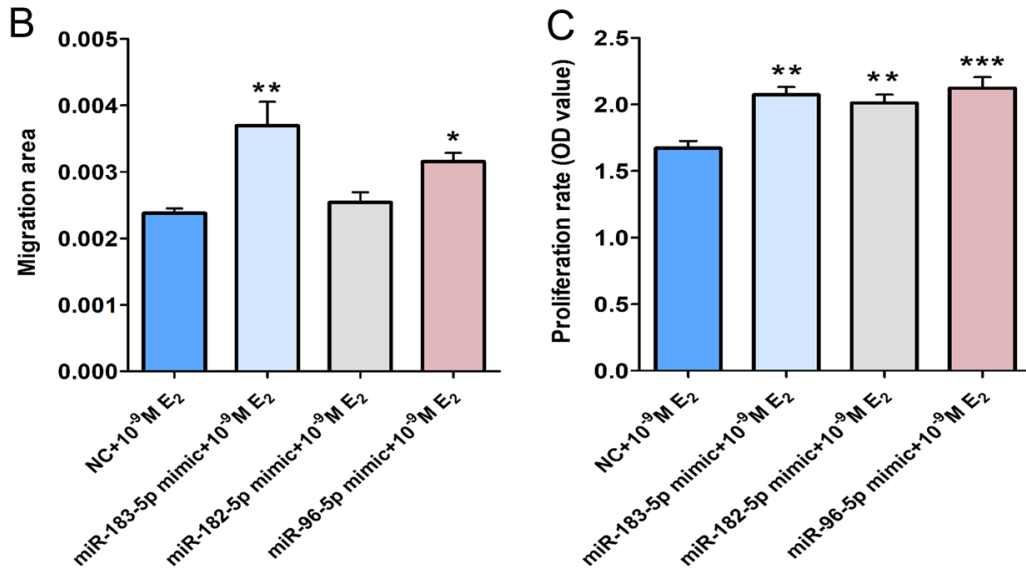

\section{Figure 3}

Effect of miR-183-5p, miR-182-5p and miR-96-5p mimics in cell migration and proliferation.

Treatment of IK cells with mimics for miR-183-5p and miR-96-5p significantly increased cell migration in the presence of $10^{-9} \mathrm{M} \mathrm{E}_{2}$ (A and $\mathrm{B}$ ). Cell proliferation also increased significantly by mimics for miR-183-5p, miR-182-5p and miR-96-5p, in the presence of $10^{-9} \mathrm{M} \mathrm{E}_{2}$ (C). $\star P<0.05$ and $* * P<0.01$, compared with corresponding controls. Number of repeated experiments $(n)=3$. activity of Renilla luciferase significantly decreased after co-transfection of the WT luciferase reporter construct and miR-183-5p-mimic, whereas no significant change was observed in enzymatic activity when co-transfection of a mutant luciferase reporter construct and miR-1835p-mimic was performed (Fig. 5C), suggesting that miR183-5p could regulate CTNNA2 expression.

To verify the regulatory role of miR-183-5p in CTNNA2 levels, we performed Western blot in IK cells transfected with I-miR-183-5p in the presence of $10^{-7} \mathrm{M} \mathrm{E}_{2}$, or miR$183-5 \mathrm{p}$-mimic in the presence of $10^{-9} \mathrm{M} \mathrm{E}_{2}$. We found that CTNNA2 was significantly upregulated by I-miR-183-5p, and, downregulated by miR-183-5p-mimic (Fig. 5D and E). Q-PCR analysis also showed that the expression levels of CTNNA2 mRNA were increased in IK cells transfected with I-miR-183-5p, and, decreased in IK cells transfected with miR-183-5p-mimic (Fig. 5G).

It has been shown that the Wnt pathway play an important role in endometrial receptivity (Tepekoy et al. 2015). GSK3 $\beta$ (Glycogen Synthase Kinase 3 Beta) is a negative regulator of Wnt/ $\beta$-catenin pathway (Ma et al. 2014, Ahmed et al. 2016, Zhang \& Yan 2016). We found that I-miR-183-5p not only upregulated CTNNA2 levels in IK cells, but also increased GSK3 $\beta$ protein and GSK3 $\beta$ mRNA levels (Fig. 5D, F and H). On the other hand, miR-1835 -mimic attenuated levels of both CTNNA2 and GSK3 $\beta$ protein and mRNA (Fig. 5D, F and H). These results suggest that the Wnt pathway might be regulated by miR-183-5p.
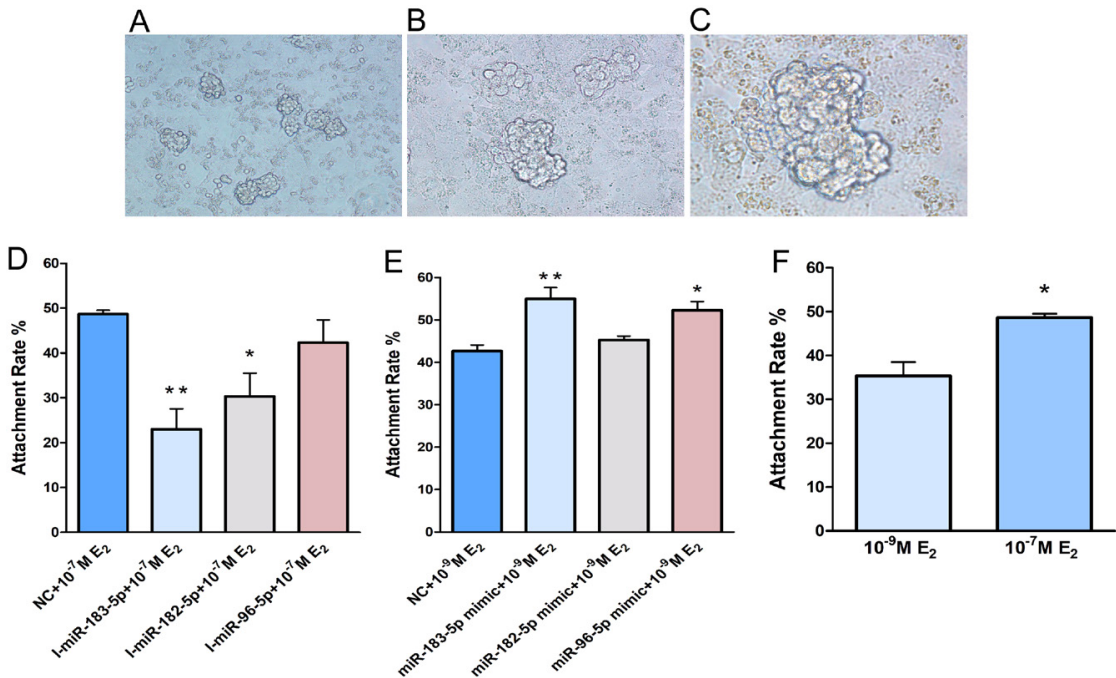

\section{Figure 4}

Effects of miR-183-5p, miR-182-5p and miR-96-5p on JAr spheroid attachment to IK cell monolayer. JAr spheroids attached to IK cells at $10 \times(A), 20 \times$ (B) and 40× (C) magnification. $\mathrm{E}_{2}$ at 10-7 M significantly increased JAr spheroid attachment rate (F). Attachment rates were significantly reduced after IK cells were treated with I-miR-183-5p and I-miR-182-5p, respectively (D), and, significantly increased after IK cells were treated with miR-183-5p-mimic and miR-96-5pmimic, respectively $(E) . * P<0.05$ and $* * P<0.01$, compared with corresponding controls. Number of repeated experiments $(n)=3$. 
A

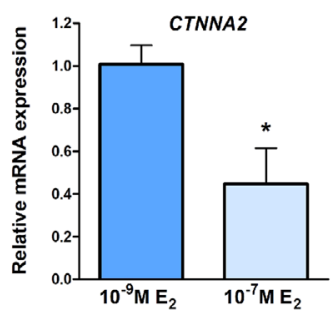

B

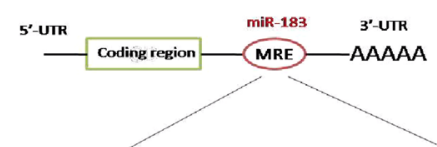

Position 597-603 of CTNNA2 3' UTR $55^{\prime}$ AGUGAAUUGUAGGU-GUGCCAU-A II I II I mir-183-5p $3^{\prime}$ ACCUACAAUUCACU-CACGGUA- $U$

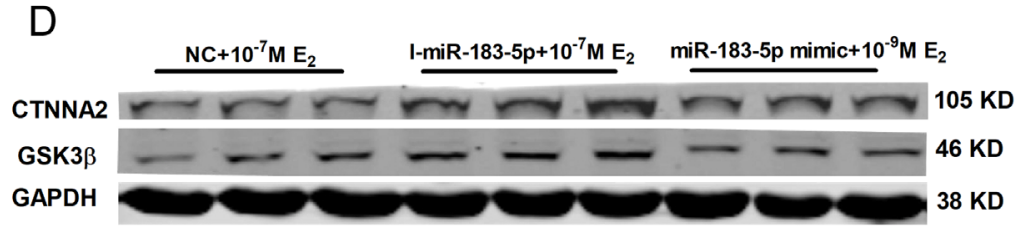

$E$

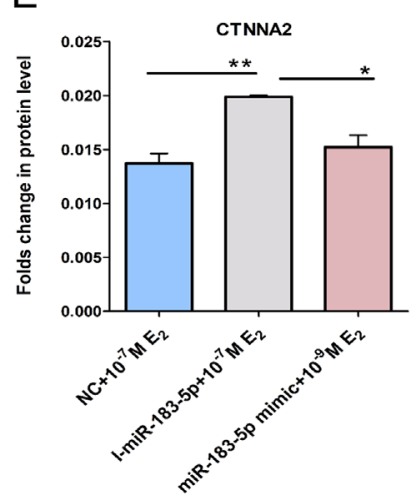

$\mathrm{F}$

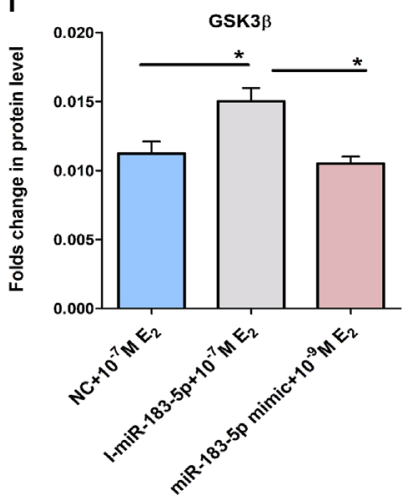

G

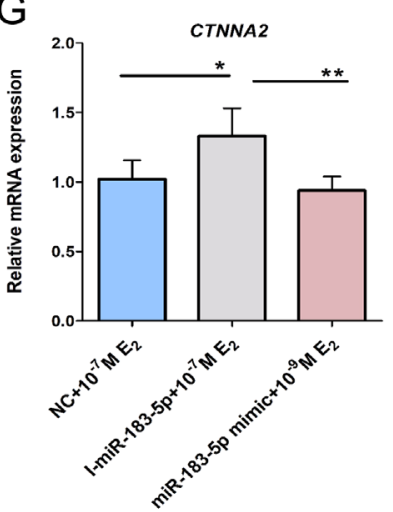

C

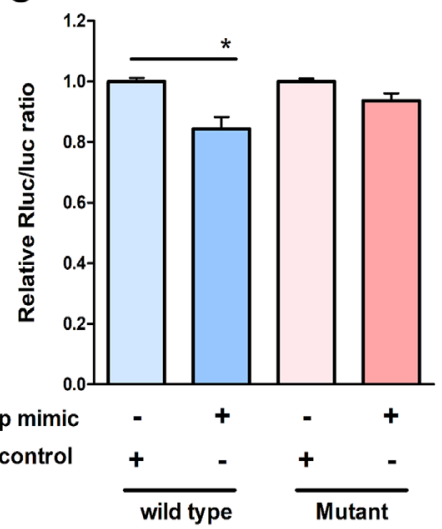

Figure 5

miR-183-5p targeting CTNNA2 was validated by luciferase reporter assay. Binding of miR-183-5p to its seed sequence within 3 'UTR of CTNNA2 mRNA (B). Renilla luciferase (Rluc) activity was significantly decreased after co-transfection of wild type CTNNA2 3'UTR and miR-183-5p mimic, while there is no significant decrease by using mutant CTNNA2 3'UTR (C). Q-PCR analysis shows that CTNNA2 mRNA expression levels in IK cells were significantly decreased by $E_{2}$ at 10-7 M (A) and miR-183-5p-mimic (G), and, increased by I-miR-183-5p (G). Western blot analyses show increased levels of CTNNA2 ( $D$ and $E$ ) and GSK3 $\beta$ (D and F) proteins in IK cells transfected with I-miR-183-5p, and, decreased levels of CTNNA2 (D and E) and GSK3 $\beta$ (D and F) proteins in IK cells transfected with miR-183-5p-mimic. GSK3 $\beta$ mRNA levels are also increased by I-miR-183-5p and decreased by miR-183-5p-mimic (H). * $P<0.05$ and $* \star P<0.01$, compared with corresponding controls. Number of repeated experiments $(n)=3$.

\section{Inhibition of miR-183-5p attenuated uterine} receptivity and embryo implantation in mouse model

We further verified roles of miR-183-5p in uterine receptivity and embryo implantation by an in vivo mouse model. Inhibitor for miR-183-5p was injected into one horn of mouse uterus while normal saline was injected into the other horn as control at day 3 of pregnancy (Fig. $6 \mathrm{~A})$. Inhibition of miR-183-5p significantly decreased embryo implantation rate in mouse dissected at day 7 of pregnancy (Fig. 6B). Meanwhile, we also found that I-miR183-5p not only inhibited the expression level of miR183-5p (Fig. 6D), but also upregulated expression levels of both CTNNA2 (Fig. 6C and E) and GSK3 $\beta$ (Fig. 6C and F) in the horn of uterus treated with miR-183-5p inhibitor as compared to the control horn. The results suggest that CTNNA2 and GSK3 $\beta$ might be involved in the regulatory role of miR-183-5p in endometrial receptivity and embryo implantation.

\section{Discussion}

The establishment of receptive endometrium is essential for successful embryo implantation. Poor endometrial receptivity is one of the major causes of implantation failure and infertility (Fatemi \& Popovic-Todorovic 2013). Currently, there is great interest in the identification of miRNAs as markers for the detection of impaired endometrial receptivity, altered embryo development and implantation failure (Nothnick 2012). Several studies examined whether miRNAs were involved in the process of uterine receptivity and embryo implantation (Chakrabarty et al. 2007, Chu et al. 2015, Zheng et al. 2017). 
A
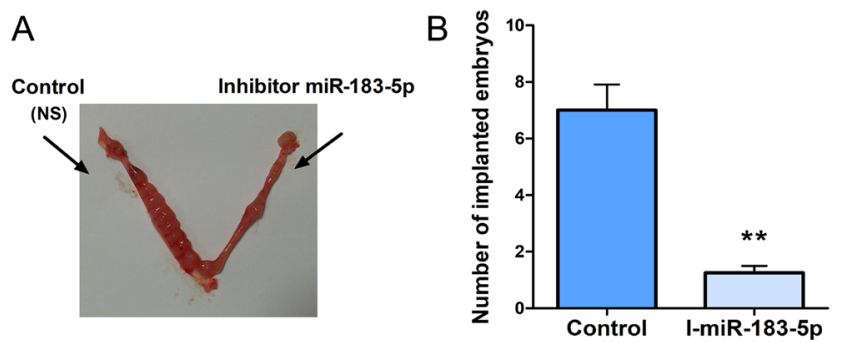

C

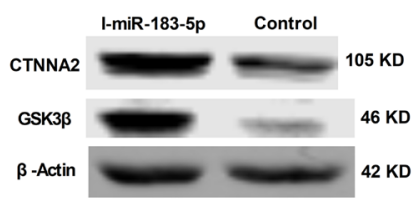

E

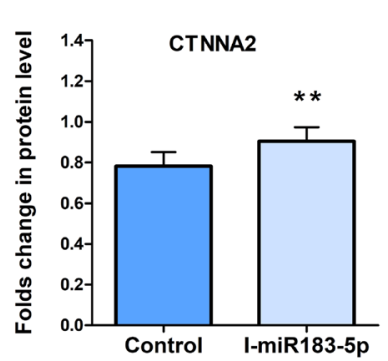

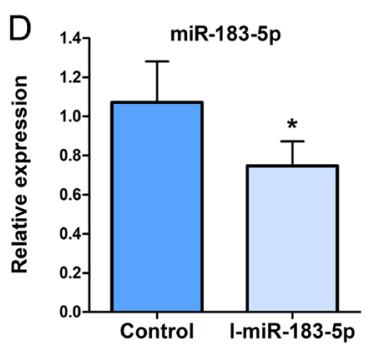



\section{Figure 6}

Mouse uterus was injected with normal saline (NS) and I-miR-183-5p at day 3 of pregnancy, and, observed on day 7 (A). Number of embryo implantation was significantly decreased in the uterine horn injected with I-miR-183-5p (B). Western blot analysis shows that levels of CTNNA2 and GSK3 $\beta$ were significantly increased in the uterine horn injected with I-miR-183-5p (C, E and F). Q-PCR analysis shows that miR-183-5p levels were also decreased in uterine horn injected with I-miR-183-5p (D). $\star P<0.05$ and $* * P<0.01$, compared with corresponding controls. Number of mouse $(n)=5$.

In the present study, we showed for the first time, the roles of the miR-183 family comprising three miRs (miR183-5p, miR-182-5p, and miR-96-5p) in endometrial receptivity and embryo implantation.

Previous studies demonstrated that miR-183 was upregulated in the endometrial fluid in the late secretory phase (Vilella et al. 2015), and, the miR-183 family was significantly downregulated during human endometrial stromal decidualization (Estella et al. 2012). Using the IK cell line, we identified that $\mathrm{E}_{2}$ significantly upregulated expression levels of the miR-183 family, and this family has positive roles in migration and proliferation of IK cells. By using in vitro JAr spheroid assay, we found that miR183-5p played a significant role in embryo attachment as inhibiting miR-183-5p considerably reduced attachment rates, and, miR-183-5p-mimic increased attachment rates.

Our in vivo mouse model also showed that inhibition of miR-183 levels results in low embryo implantation rates.

We evaluated potential target genes of the miRs mentioned above by using mi-RNA target predicting software. The identified genes (CTNNA2, LRP6, KCNJ14, HOXA9, and FGF-9) have been reported to be downregulated in the secretory phase of the female reproductive cycle in different studies (Carson et al. 2002, Horcajadas et al. 2006, Díaz-Gimeno et al. 2011). Luciferase reporter assay in the present study validated that CTNNA2 gene is a potential target gene of miR-183-5p.

The complexity of tight junctions significantly reduce in receptive-phase endometrium, and, less complex tight junctions may assist penetration of blastocyst and endometrium (Murphy et al. 1992). Another study demonstrated that components of gap junctions and adhesion decreased during the pre-implantation period to facilitate trophoblast invasion through uterine epithelium (Grund \& Grümmer 2018). Alpha-catenin also regulates actin dynamics, negatively correlating cell adhesion with cell proliferation (Kobielak \& Fuchs 2004). Activation of Wnt/ $\beta$-catenin pathway increases uterine receptivity and adhesion of embryo and endometrial cells (Zheng et al. 2017). CTNNA2 is responsible for cell-cell adhesion by maintaining the integrity of the cadherin-catenin complex and linking adherent junctions to the actin cytoskeleton (Gates \& Peifer 2005). The present study showed that miR183-5p may increase cell migration and proliferation of endometrial cells and downregulate expression levels of CTNNA2 and GSK3 $\beta$ that is a negative regulator of $\mathrm{Wnt} / \beta$ catenin pathway (Ma et al. 2014, Ahmed et al. 2016, Zhang \& Yan 2016). Our data indicate that miR-183-5p might be involved in promoting the effects of estrogen on endometrial receptivity and embryo implantation by decreasing endometrial cell-cell adhesion, and, the junctional complexity of uterine epithelial cells.

Our in vivo mouse model showed that inhibition of miR-183 levels resulted in low embryo implantation rate, and, higher CTNNA2 and GSK3 $\beta$ levels in uterus. Recently, it was reported that the inactivation of Wnt/ $\beta$ catenin signaling impaired receptive uterine endometrium formation (Zheng et al. 2017). We proposed a hypothetical model showing how miR-183-5p regulates the CTNNA2 gene and results in downstream changes, which can ultimately affect the receptivity of endometrium (Fig. 7).

Embryonic implantation in humans depends on the interaction of the embryo with the receptive endometrium. The roles of $\mathrm{E}_{2}$ in embryo implantation are contradictory in different studies. High levels of estrogen can be observed in women during in vitro fertilization 


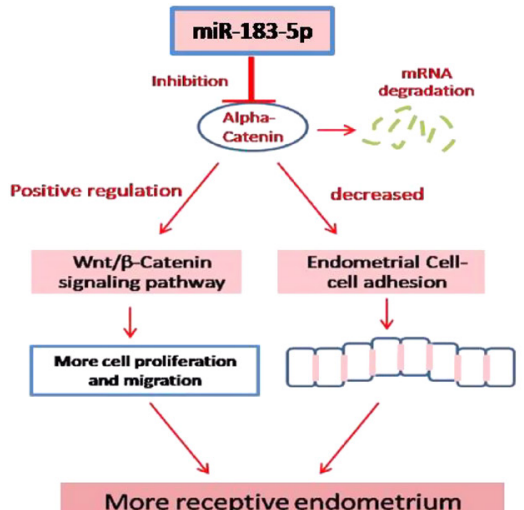

More receptive endometrium

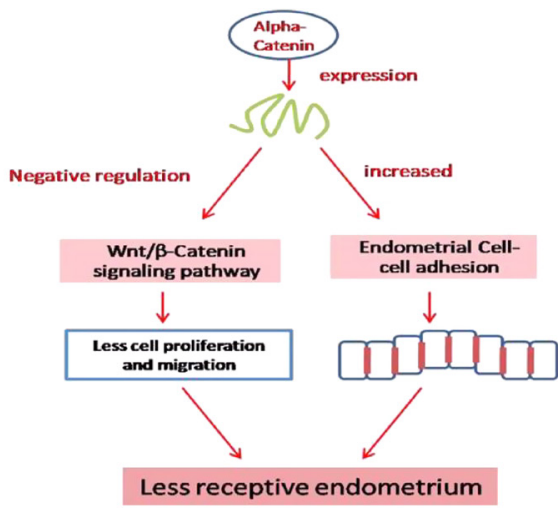

\section{Figure 7}

Hypothetical model shows the role of miR-183-5p in regulating endometrial receptivity via targeting CTNNA2 gene. and embryo transfer (IVF-ET), and, high doses of estrogen are also used in endometrium preparation in patients underlying frozen embryo implantation (Berkane et al. 2017). Several studies demonstrated that supraphysiological $\mathrm{E}_{2}$ levels were not detrimental to IVF-ET outcomes (Jing et al. 2019, Sekhon et al. 2019), although previous studies suggested that exposure to high levels of $\mathrm{E}_{2}$ during IVF treatment in the early follicular phase might be related to a lower chance of pregnancy and IVF outcome (Kolibianakis et al. 2003). Our previous study and the present studies also showed that $10^{-7} \mathrm{M}$ $\mathrm{E}_{2}$ promoted JAr spheroid attachment on IK cells (Ullah et al. 2017).

In the present study, we demonstrated a part of the roles and underlying mechanisms of miR-183 family in uterine receptivity and embryo implantation. The roles of other potential target genes of miR-183 family involved in the process of endometrial receptivity and embryo implantation may be important for the clinical application of the miR-183 family as a biomarker for implantation failures, and, new strategy for infertility treatment.

\section{Supplementary materials}

This is linked to the online version of the paper at https://doi.org/10.1530/ JME-19-0184.

\section{Declaration of interest}

The authors declare that there is no conflict of interest that could be perceived as prejudicing the impartiality of the research reported.

\section{Funding}

This study was supported by the National Key Research and Development Program of China (No. 2017 YFC1001001 and No. 2018YFC1004402 to J-Z S), the National Natural Science Foundation of China (No. 31671569 to J-Z S) and the Special Fund for the National Key Research and Development Plan Grant (No. 2017 YFC1001300 to H-F H).

(C) 2020 Society for Endocrinology Published by Bioscientifica Ltd. Printed in Great Britain

\section{Author contribution statement}

RA, KU and J-ZS designed the experiments. RA performed the experiments, T U R helped to analyze the data. L-Y J, Y C, H-Y P and Q-J W helped in performing experiments. $\mathrm{R} \mathrm{A}, \mathrm{H}-\mathrm{F} \mathrm{H}$ and J-Z $\mathrm{S}$ wrote and edited the paper.

\section{Acknowledgments}

All authors thank Dr M J Quinn from U.K. for his reading and editing this manuscript.

\section{References}

Abbassi-Ghanavati M, Greer LG \& Cunningham FG 2009 Pregnancy and laboratory studies: a reference table for clinicians. Obstetrics and Gynecology 114 1326-1331. (https://doi.org/10.1097/ AOG.0b013e3181c2bde8)

Achache H \& Revel A 2006 Endometrial receptivity markers, the journey to successful embryo implantation. Human Reproduction Update 12 731-746. (https://doi.org/10.1093/humupd/dml004)

Ahmed RA, Alawin OA \& Sylvester PW $2016 \gamma$-Tocotrienol reversal of epithelial-to-mesenchymal transition in human breast cancer cells is associated with inhibition of canonical Wnt signalling. Cell Proliferation 49 460-470. (https://doi.org/10.1111/cpr.12270)

Bai L, Wang H, Wang AH, Zhang LY \& Bai J 2017 MicroRNA-532 and microRNA-3064 inhibit cell proliferation and invasion by acting as direct regulators of human telomerase reverse transcriptase in ovarian cancer. PLOS ONE 12 e0173912. (https://doi.org/10.1371/ journal.pone.0173912)

Bartel DP 2004 MicroRNAs: genomics, biogenesis, mechanism, and function. Cell 116 281-297. (https://doi.org/10.1016/s00928674(04)00045-5)

Berkane N, Liere P, Oudinet JP, Hertig A, Lefèvre G, Pluchino N, Schumacher M \& Chabbert-Buffet N 2017 From pregnancy to preeclampsia: a key role for estrogens. Endocrine Reviews 38 123-144. (https://doi.org/10.1210/er.2016-1065)

Carson DD, Lagow E, Thathiah A, Al-Shami R, Farach-Carson MC, Vernon M, Yuan L, Fritz MA \& Lessey B 2002 Changes in gene expression during the early to mid-luteal (receptive phase) transition in human endometrium detected by high-density microarray screening. Molecular Human Reproduction 8 871-879. (https://doi. org/10.1093/molehr/8.9.871)

Chakrabarty A, Tranguch S, Daikoku T, Jensen K, Furneaux H \& Dey SK 2007 MicroRNA regulation of cyclooxygenase-2 during embryo implantation. PNAS 104 15144-15149. (https://doi.org/10.1073/ pnas.0705917104)

Chu B, Zhong L, Dou S, Wang J, Li J, Wang M, Shi Q, Mei Y \& Wu M 2015 miRNA-181 regulates embryo implantation in mice through 
targeting leukemia inhibitory factor. Journal of Molecular Cell Biology 7 12-22. (https://doi.org/10.1093/jmcb/mjv006)

Díaz-Gimeno P, Horcajadas JA, Martínez-Conejero JA, Esteban FJ, Alamá P, Pellicer A \& Simón C 2011 A genomic diagnostic tool for human endometrial receptivity based on the transcriptomic signature. Fertility and Sterility 95 50.e1-60.e1. (https://doi. org/10.1016/j.fertnstert.2010.04.063)

Estella C, Herrer I, Moreno-Moya JM, Quiñonero A, Martínez S, Pellicer A \& Simón C 2012 miRNA signature and Dicer requirement during human endometrial stromal decidualization in vitro. PLoS ONE 7 e41080. (https://doi.org/10.1371/journal.pone.0041080)

Fatemi HM \& Popovic-Todorovic B 2013 Implantation in assisted reproduction: a look at endometrial receptivity. Reproductive Biomedicine Online 27 530-538. (https://doi.org/10.1016/j. rbmo.2013.05.018)

Gao Q, Gu Y, Jiang Y, Fan L, Wei Z, Jin H, Yang X, Wang L, Li X, Tai S, et al. 2018 Long non-coding RNA Gm2199 rescues liver injury and promotes hepatocyte proliferation through the upregulation of ERK1/2. Cell Death and Disease 9 602. (https://doi.org/10.1038/ s41419-018-0595-9)

Gates J \& Peifer M 2005 Can 1000 reviews be wrong? Actin, $\alpha$-catenin, and adherens junctions. Cell 123 769-772. (https://doi. org/10.1016/j.cell.2005.11.009)

Grund S \& Grümmer R 2018 Direct cell-cell interactions in the endometrium and in endometrial pathophysiology. International Journal of Molecular Sciences 19 2227. (https://doi.org/10.3390/ ijms19082227)

Ha M \& Kim VN 2014 Regulation of microRNA biogenesis. Nature Reviews: Molecular Cell Biology 15 509-524. (https://doi.org/10.1038/ nrm3838)

Hohn HP, Linke M \& Denker HW 2000 Adhesion of trophoblast to uterine epithelium as related to the state of trophoblast differentiation: in vitro studies using cell lines. Molecular Reproduction and Development 57 135-145. (https://doi.org/10.1002/10982795(200010) 57:2<135::AID-MRD4>3.0.CO;2-9)

Horcajadas JA, Pellicer A \& Simon C 2006 Wide genomic analysis of human endometrial receptivity: new times, new opportunities. Human Reproduction Update 13 77-86. (https://doi.org/10.1093/ humupd/dml046)

Jing Z, Xi H, Qianling Z, Lunquan S, Nenghui L \& Yanping L 2019 Oestrogen dose tapering during luteal phase does not affect clinical outcomes after hormone replacement treatment-frozen-thawed embryo transfer cycles: a retrospective analysis. Human Reproduction 34 1479-1484. (https://doi.org/10.1093/humrep/dez096)

Kobielak A \& Fuchs E $2004 \alpha$-Catenin: at the junction of intercellular adhesion and actin dynamics. Nature Reviews: Molecular Cell Biology 5 614-625. (https://doi.org/10.1038/nrm1433)

Kolibianakis EM, Albano C, Kahn J, Camus M, Tournaye H, Van Steirteghem AC \& Devroey P 2003 Exposure to high levels of luteinizing hormone and estradiol in the early follicular phase of gonadotropin-releasing hormone antagonist cycles is associated with a reduced chance of pregnancy. Fertility and Sterility 79 873-880. (https://doi.org/10.1016/s0015-0282(02)04920-8)

Liang J, Wang S \& Wang Z 2017 Role of microRNAs in embryo implantation. Reproductive Biology and Endocrinology 15 90. (https:// doi.org/10.1186/s12958-017-0309-7)
Ma MZ, Zhuang C, Yang XM, Zhang ZZ, Ma H, Zhang WM, You H, Qin W, Gu J, Yang S, et al. 2014 CTHRC1 acts as a prognostic factor and promotes invasiveness of gastrointestinal stromal tumors by activating Wnt/PCP-Rho signaling. Neoplasia 16 265.e1-278.e1. (https://doi.org/10.1016/j.neo.2014.03.001)

Murphy CR, Rogers PA, Hosie MJ, Leeton J \& Beaton L 1992 Tight junctions of human uterine epithelial cells change during the menstrual cycle: a morphometric study. Acta Anatomica 144 36-38. (https://doi.org/10.1159/000147282)

Nothnick WB 2012 The role of microRNAs in the female reproductive tract. Reproduction 143 559-576. (https://doi.org/10.1530/REP-110240)

Sekhon L, Feuerstein J, Pan S, Overbey J, Lee JA, Briton-Jones C, Flisser E, Stein DE, Mukherjee T, Grunfeld L, et al. 2019 Endometrial preparation before the transfer of single, vitrified-warmed, euploid blastocysts: does the duration of estradiol treatment influence clinical outcome? Fertility and Sterility 111 1177.e3-1185.e3. (https:// doi.org/10.1016/j.fertnstert.2019.02.024)

Strowitzki T, Germeyer A, Popovici R \& Von Wolff M 2006 The human endometrium as a fertility-determining factor. Human Reproduction Update 12 617-630. (https://doi.org/10.1093/humupd/dml033)

Sun Y, Zhang J \& Ma L $2014 \alpha$-Catenin: a suppressor beyond adherens junctions. Cell Cycle 13 2334-2339. (https://doi.org/10.4161/ cc.29765)

Tepekoy F, Akkoyunlu G \& Demir R 2015 The role of Wnt signaling members in the uterus and embryo during pre-implantation and implantation. Journal of Assisted Reproduction and Genetics 32 337-346. (https://doi.org/10.1007/s10815-014-0409-7)

Ullah K, Rahman TU, Pan HT, Guo MX, Dong XY, Liu J, Jin LY, Cheng Y, Ke ZH, Ren J, et al. 2017 Serum estradiol levels in controlled ovarian stimulation directly affect the endometrium. Journal of Molecular Endocrinology 59 105-119. (https://doi. org/10.1530/JME-17-0036)

Vilella F, Moreno-Moya JM, Balaguer N, Grasso A, Herrero M, Martínez S, Marcilla A \& Simón C 2015 Hsa-miR-30d, secreted by the human endometrium, is taken up by the pre-implantation embryo and might modify its transcriptome. Development 142 3210-3221. (https://doi.org/10.1242/dev.124289)

Yang P, Wu Z, Pan N, Wang Y \& Yan L 2018 Endometrial miR-543 is downregulated during the implantation window in women with endometriosis-related infertility. Reproductive Sciences 26 900-908. (https://doi.org/10.1177/1933719118799199)

Zhang Q \& Yan J 2016 Update of Wnt signaling in implantation and decidualization. Reproductive Medicine and Biology 15 95-105. (https:// doi.org/10.1007/s12522-015-0226-4)

Zheng Q, Zhang D, Yang YU, Cui X, Sun J, Liang C, Qin H, Yang X, Liu S \& Yan Q 2017 MicroRNA-200c impairs uterine receptivity formation by targeting FUT4 and $\alpha 1,3$-fucosylation. Cell Death and Differentiation 24 2161-2172. (https://doi.org/10.1038/ cdd.2017.136)

Zou LB, Zhang RJ, Tan YJ, Ding GL, Shi S, Zhang D, He RH, Liu AX, Wang TT, Leung PC, et al. 2011 Identification of estrogen response element in the aquaporin-2 gene that mediates estrogen-induced cell migration and invasion in human endometrial carcinoma. Journal of Clinical Endocrinology and Metabolism 96 E1399-E1408. (https://doi. org/10.1210/jc.2011-0426)

Received in final form 20 November 2019

Accepted 27 November 2019

Accepted Manuscript published online 27 November 2019 https://jme.bioscientifica.com https://doi.org/10.1530/JME-19-0184 (c) 2020 Society for Endocrinology Published by Bioscientifica Ltd. Printed in Great Britain 